\title{
FURTHER ELEMENTARY TECHNIQUES USING THE MIRACLE OCTAD GENERATOR
}

\author{
by R. T. CURTIS
}

(Received 20th July 1987)

\section{Introduction}

In this paper we describe various techniques, some of which are already used by devotees of the art, which relate certain maximal subgroups of the Mathieu group $M_{24}$, as seen in the MOG, to matrix groups over finite fields. We hope to bring out the wealth of algebraic structure underlying the device and to enable the reader to move freely between these matrices and permutations. Perhaps the MOG was mis-named as simply an "octad generator"; in this paper we intend to show that it is in reality a natural diagram of the binary Golay code.

There are two versions of the MOG in print: the author's original version which appears in $[5,6,7,8]$, and what is, in effect, the mirror-image of this which is used in $[1,3,4,10]$. Certain subgroups "look better" in each system and so it is worthwhile having both arrangements available. We shall indicate which we are referring to at any point by appending the subscripts $M$ and $M^{\prime}$ respectively.

The octads in each case are, of course, those listed in [9].

N.B. To go from $M$ to $M^{\prime}$, or vice versa, take the mirror-image or equivalently (modulo $M_{24}$ ) interchange the last two columns.

\section{Mnemonic for obtaining a MOG arrangement of the 24 letters (John Conway)}

(1) Insert the first twelve members of the Galois field of order 23 in a $4 \times 6$ array as shown in Fig. 1.

(2) Negate the non-squares (Fig. 2).

(3) Fill in the remainder of the 24-point projective line letting the linear fractional map

$$
\begin{aligned}
& \gamma: x \rightarrow-1 / x \equiv(\infty \quad 0)(1 \quad 22)(2 \quad 11)(3 \quad 15)\left(4 \begin{array}{lll}
2 & 17
\end{array}\right)\left(\begin{array}{lll}
5 & 9
\end{array}\right) \\
& \left(\begin{array}{llll}
6 & 19
\end{array}\right)(7 \quad 13)(8 \quad 20)\left(\begin{array}{lll}
10 & 16
\end{array}\right)(12 \quad 21)\left(\begin{array}{ll}
14 & 18
\end{array}\right)
\end{aligned}
$$

\begin{tabular}{|c|c|c|}
\hline 30 & 4 & $5^{2}$ \\
\hline 6 & 7 & 8 \\
\hline 9 & 10 & 11 \\
\hline
\end{tabular}

correspond to the permutation indicated in Fig. 3.

FIGURE 1

\begin{tabular}{|c|c|c|c|}
\hline 3 & & & 1 \\
\hline 6 & 16 & 8 \\
9 & 13 & 12 \\
\hline
\end{tabular}

FIGURE 2



FIGURE 3 
(4) Obtain

\begin{tabular}{|rr|rr|rr|}
\hline$\infty$ & 0 & 11 & 1 & 22 & 2 \\
3 & 19 & 4 & 20 & 18 & 10 \\
\hline 6 & 15 & 16 & 14 & 8 & 17 \\
9 & 5 & 13 & 21 & 12 & 7 \\
\hline
\end{tabular}

\begin{tabular}{|rr|rr|rr|}
\hline 0 & $\infty$ & 1 & 11 & 2 & 22 \\
19 & 3 & 20 & 4 & 10 & 18 \\
\hline 15 & 6 & 14 & 16 & 17 & 8 \\
5 & 9 & 21 & 13 & 7 & 12 \\
\hline
\end{tabular}

FIGURE 4

A. $M_{21} \cong L_{3}(4)$

A.1. We take the three fixed points as those indicated by $X$ 's in Fig. 5 and the remaining five points of the left-hand brick as the line at infinity, $z=0$. The 16-point affine plane is co-ordinated with $x$-axis horizontal (left to right) and $y$-axis vertical (descending) so that the projective point $(001)^{t}$ appears in the top lefthand position.

\begin{tabular}{|c|c|c|c|c|c|}
\hline$\left(\begin{array}{l}0 \\
1 \\
0\end{array}\right)$ & $\left(\begin{array}{l}1 \\
0 \\
0\end{array}\right)$ & $\left(\begin{array}{l}0 \\
0 \\
1\end{array}\right)$ & $\left(\begin{array}{l}1 \\
0 \\
1\end{array}\right)$ & $\left(\begin{array}{l}\omega \\
0 \\
1\end{array}\right)$ & $\left(\begin{array}{l}\omega \\
0 \\
1\end{array}\right.$ \\
\hline$x$ & $\left(\begin{array}{l}1 \\
1 \\
0\end{array}\right)$ & $\left(\begin{array}{l}0 \\
1 \\
1\end{array}\right)$ & $\left(\begin{array}{l}1 \\
1 \\
1\end{array}\right)$ & $\left(\begin{array}{l}w \\
1 \\
1\end{array}\right)$ & $\left(\begin{array}{l}\omega \\
1 \\
1\end{array}\right)$ \\
\hline$x$ & $\left(\begin{array}{l}1 \\
\omega \\
0\end{array}\right)$ & $\left(\begin{array}{l}0 \\
\omega \\
1\end{array}\right)$ & $\left(\begin{array}{l}1 \\
\omega \\
1\end{array}\right)$ & $\left(\begin{array}{l}\omega \\
\omega \\
1\end{array}\right)$ & {$\left[\begin{array}{l}\omega \\
\omega \\
1\end{array}\right.$} \\
\hline$x$ & $\left(\begin{array}{l}\frac{1}{\omega} \\
0\end{array}\right)$ & $\left(\begin{array}{l}\frac{0}{\omega} \\
1\end{array}\right)$ & $\left(\begin{array}{l}\frac{1}{\omega} \\
1\end{array}\right)$ & $\left(\begin{array}{l}\frac{\omega}{\omega} \\
1\end{array}\right)$ & {$\left[\begin{array}{l}\frac{\omega}{\omega} \\
1\end{array}\right]$} \\
\hline
\end{tabular}

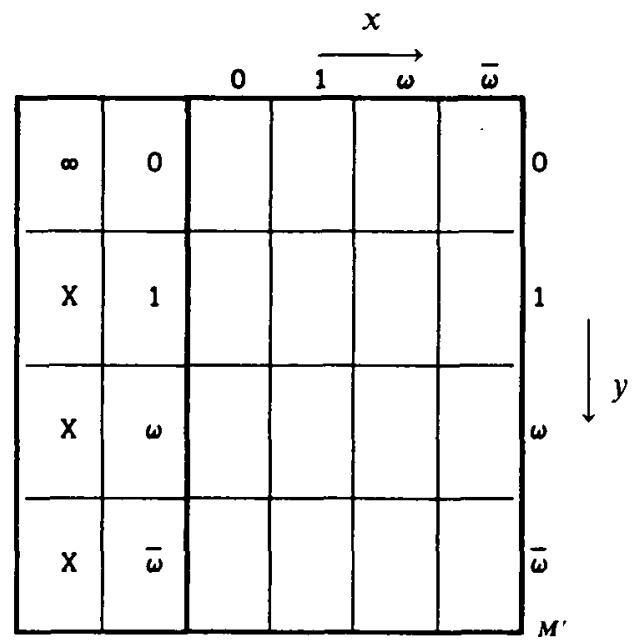

FIGURE 5

The 21-point projective plane as it appears in the MOG

Notes:

(i) The 21 octads containing the three fixed points have convenient names as lines $y=m x+b$.

e.g. $y=\omega x+1 \equiv$



(ii) A pleasing construction of the Golay code and, hence, of $M_{24}$ can be furnished straight from here. 
A.2. Identification of permutations of $M_{21}$ with matrices (modulo the centre of $S_{3}(4)$ )

A matrix is determined (up to multiplication by a scalar matrix) by its action on the triangle of reference $\left((100)^{t},(010)^{t},(001)^{t}\right)$ and the unit point $(111)^{t}$. Thus



$$
\left\{\left[\begin{array}{ccc}
1 & \cdot & \alpha \\
. & 1 & \beta \\
& . & 1
\end{array}\right] ; \alpha, \beta \in G F_{4}\right\}=\begin{aligned}
& \text { the elementary abelian } 2^{4} \text { fixing each } \\
& \text { point on the line at infinity. }
\end{aligned}
$$

Again

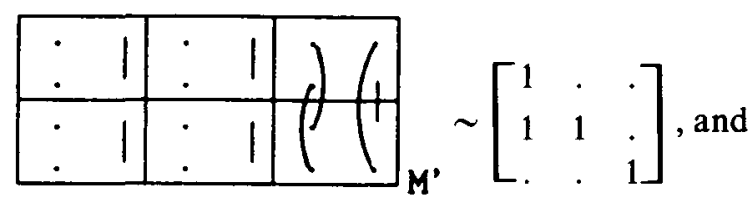

$$
\left\{\left[\begin{array}{c|c}
A & \cdot \\
\cdot & 0
\end{array}\right] ; A \in L_{2}(4)\right\}=\text { the } A_{5} \text { acting on the line at infinity fixing }(001)^{t} \text {. }
$$

Adjoining

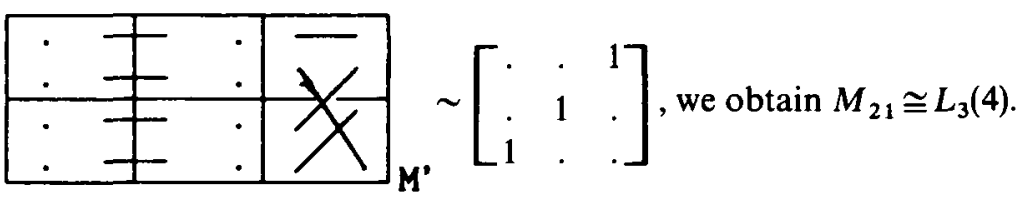

A.3. The automorphisms of $M_{21}, M_{21}: S_{3}$

(i) The field automorphism of $L_{3}(4)$ corresponds to



(ii) The diagonal automorphism of $L_{3}(4)$ may be taken as

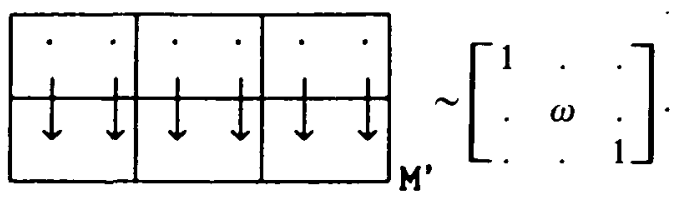

These, together with $M_{21}$, generate the maximal subgroup $M_{21}: S_{3}$ of $M_{24}$.

Note that the graph automorphism of $L_{3}(4)$, which interchanges points and lines, cannot be realised here. 
B. The stabilizer of an octad $\left(2^{4}: A_{8}=2^{4}: L_{4}(2)\right)$

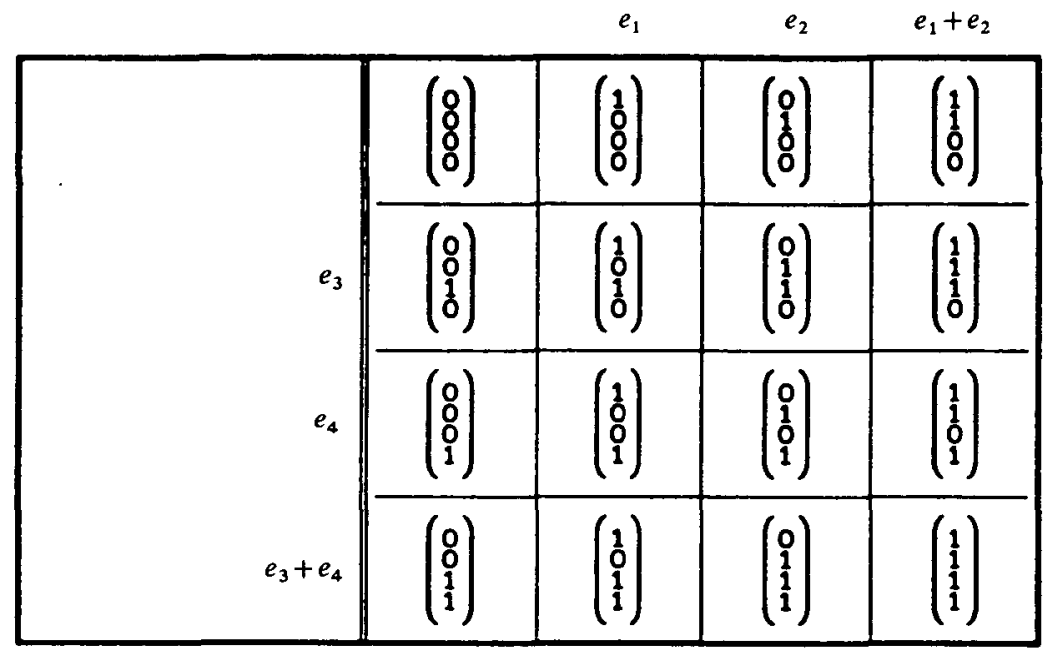

FIGURE 6

Label points in the complementary 16-ad as 4-dimensional vectors over $G F_{2}$ to give the 16-point affine plane. The stabilizer of an octad is now the semi-direct product of the elementary abelian group of translations generated by addition of a vector by the matrix group $L_{4}(2)$. This semi-direct product may be written as $5 \times 5$ matrices in the standard way. We have the following identification:

\section{B.2. Identification of permutations with matrices in $L_{\mathbf{5}}(2)$}
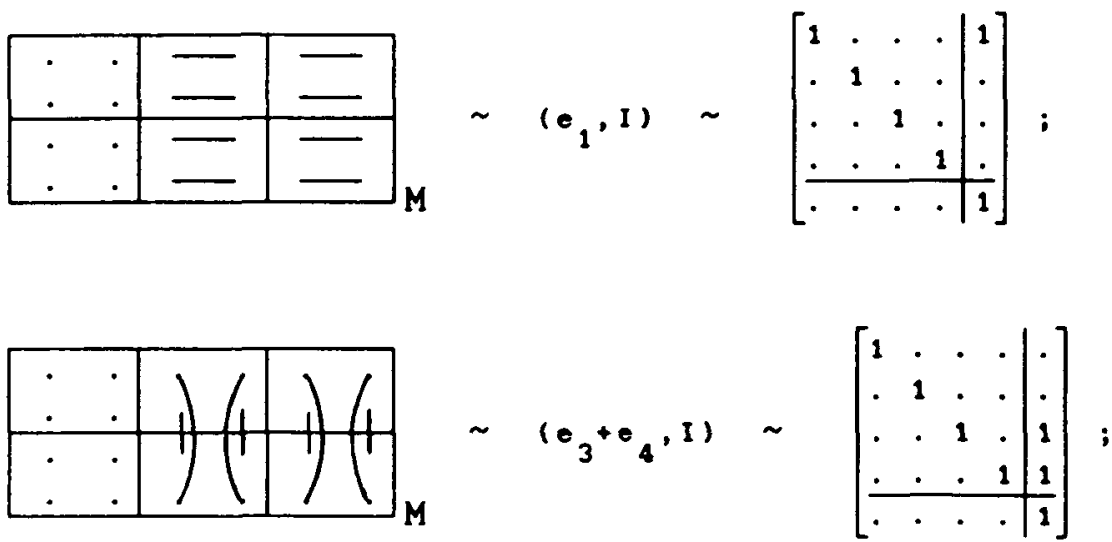

and similarly

$$
\left\{\left[\underline{I_{4}} \mid \underline{\underline{v}}\right] ; \underline{v} \in V_{4}\right\}=\begin{aligned}
& \text { the elementary abelian group of order } 16 \\
& \text { fixing every point of the octad. }
\end{aligned}
$$


The subgroup fixing the zero vector acts as $A_{8}$ on the 8 points of the octad and as $L_{4}(2)$ on the 15 non-zero vectors. Thus the MOG furnishes the isomorphism:
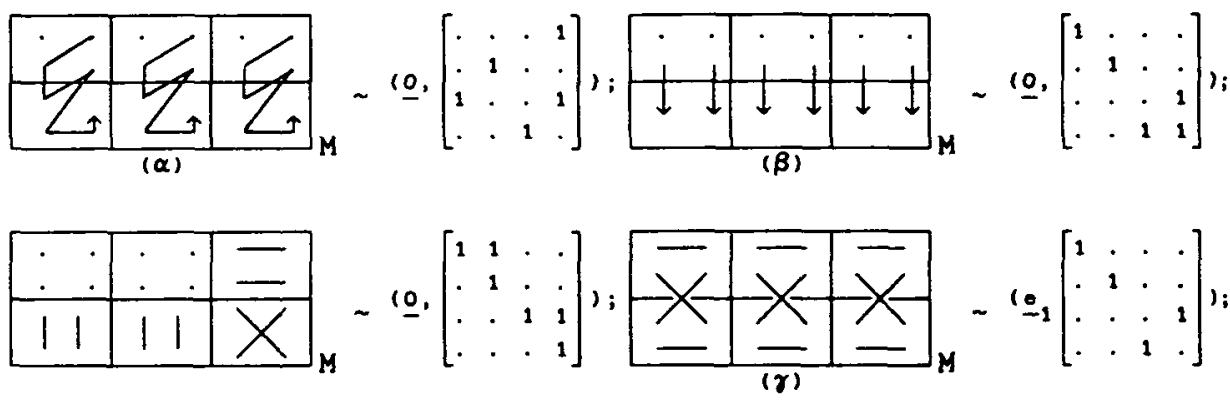

Plainly a Sylow 2-subgroup of $M_{24}$ may be taken as the non-singular upper triangular matrices in this identification.

\section{B.3. A related maximal subgroup-the trio group}

It will be noted that the elements $\alpha, \beta, \gamma$ act identically on the three bricks and, with the labelling

\begin{tabular}{|ll|}
\hline$\infty$ & 0 \\
1 & 3 \\
\hline 2 & 6 \\
4 & 5 \\
\hline
\end{tabular}

may be seen to generate $P S L_{2}(7)$ with $\alpha: x \rightarrow x+1, \beta: x \rightarrow 2 x, \gamma: x \rightarrow-1 / x$. As was pointed out in [2] the permutation $(\infty \quad 0)\left(\begin{array}{llll}1 & 3\end{array}\right)\left(\begin{array}{ll}2 & 6\end{array}\right)\left(\begin{array}{ll}4 & 5\end{array}\right)$ and its 7 images under $L_{2}(7)$, together with the identity permutation, form an elementary abelian group of order $2^{3}$. If we place an element of this group in each of the three bricks but with the restriction that the product of the three be the identity, we obtain an elementary abelian group of order $2^{6}$. This, together with the above-mentioned $L_{2}(7)$ and the $S_{3}$ bodily permuting the bricks, gives the maximal trio group of shape $2^{6}:\left(S_{3} \times L_{2}(7)\right)$.

\section{B.4. The centralizer of an involution}

The centralizer of a central $\left(1^{8} .2^{8}\right)$ involution, which we may take to be $\left(e_{1}, 1\right)$, will consist of all non-singular matrices of the form

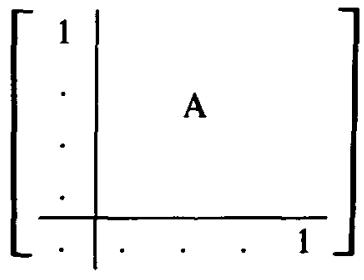


It has shape $2.2^{3+3}: L_{3}(2)$ where the two elementary abelian groups of order $16\left(2^{1+3}\right)$ consist of matrices of the form



The $L_{3}(2)$ may be taken to be those matrices of the form

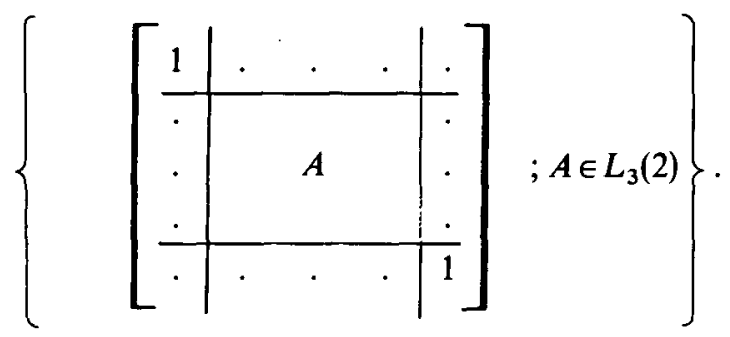

Clearly the upper uni-triangular matrices, give a Sylow 2-subgroup of $M_{24}$.

C. The sextet stabilizer, the hexacode and the Sylow 2-subgroup of $M_{\mathbf{2 4}}$

( $M$ or $M^{\prime}$ are equally good here; we choose $M^{\prime}$ to be consistent with [4]).

C.1. The stabilizer of a decomposition into 6 mutually complementary tetrads [9] or a sextet $[2,6]$ is a maximal subgroup of shape $2^{6}: 3 \cdot S_{6}$, and an example is the subgroup preserving the columns of the MOG. The permutations in the normal subgroup $2^{6}$ fix all the columns and have a four-group action on them. We denote these actions:

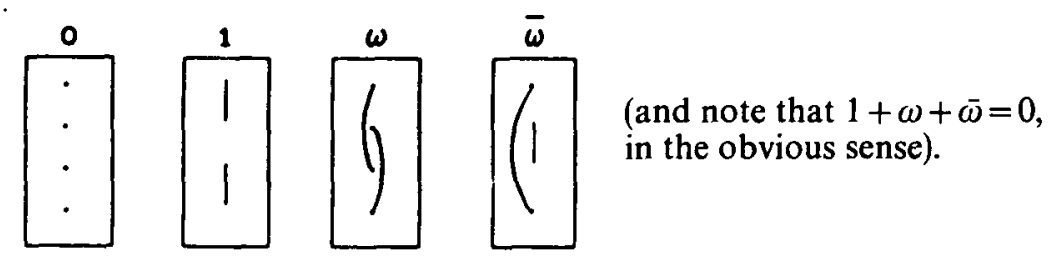

The elements of the $2^{6}$ now become 6-dimensional vectors over $G F_{4}$ and clearly form a 3-dimensional subspace, We take as basis:

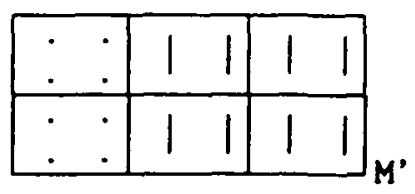

$\left(\begin{array}{llllll}0 & 0 & 1 & 1 & 1 & 1\end{array}\right)$ e

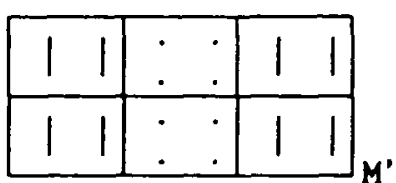

$\left(\begin{array}{llllll}1 & 1 & 0 & 0 & 1 & 1\end{array}\right)$ $e_{2}$

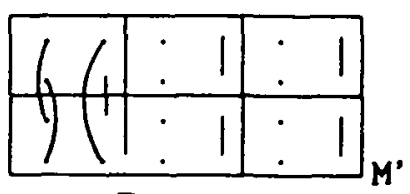

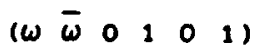

$e_{3}$ 
As can be readily verified from their expressions as 6-dimensional vectors over $G F_{4}$, 45 of the involutions of the elementary abelian $2^{6}$ have cycle shape $1^{8} \cdot 2^{8}$ while the remaining 18 have cycle shape $2^{12}$. These 18 fall into 6 blocks of size three under multiplication by $\omega$; that is they consist of the non-trivial elements of 6 disjoint fourgroups which, of course, correspond to 1-dimensional subspaces over $G F_{4}$. A set of generators for the six 1-spaces is:

$$
\left\{\left(\begin{array}{c}
1 \\
\omega \\
\omega
\end{array}\right),\left(\begin{array}{c}
1 \\
\bar{\omega} \\
\bar{\omega}
\end{array}\right),\left(\begin{array}{c}
\omega \\
\bar{\omega} \\
0
\end{array}\right),\left(\begin{array}{c}
\omega \\
\omega \\
0
\end{array}\right),\left(\begin{array}{c}
\omega \\
0 \\
\bar{\omega}
\end{array}\right),\left(\begin{array}{c}
\omega \\
0 \\
\omega
\end{array}\right)\right\} .
$$

As usual the most pleasing outcome occurs as our $S_{6}$ realizes both its 6-point actions: one on the six tetrads of the sextet, the other on these six four-groups. Thus the stabilizer of a four-group remains transitive on the tetrads and vice versa.

\section{C.2. Identification of permutations with elements of $P \Sigma L_{3}(4)$}

Now the subgroup $3 \cdot S_{6}$, which consists of permutations preserving the columns and fixing the top row of the MOG, acts as linear transformations (together with the field automorphism) of this space. In particular we see:

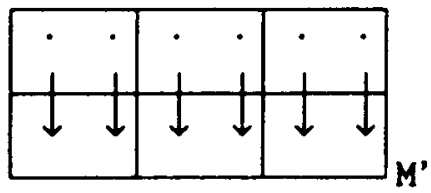

$$
\sim\left[\begin{array}{lll}
1 & \cdot & \cdot \\
\cdot & \omega & \cdot \\
\cdot & \cdot & 1
\end{array}\right] \text {. }
$$

and



Further the elements:
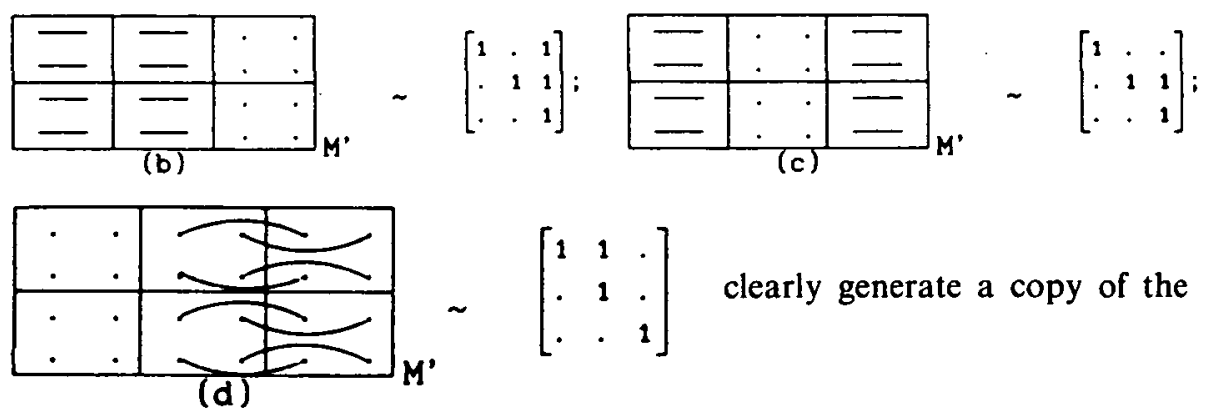

dihedral group $D_{8}$. 
We also observe that

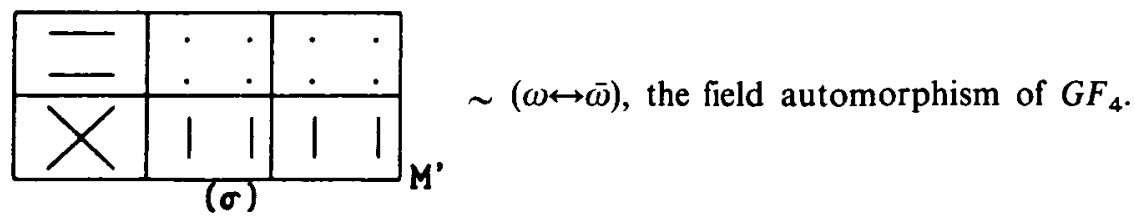

It should be noted that $\langle a, b\rangle \cong 3 \cdot A_{6}$, the triple cover of $A_{6}$.

\section{C.3. The Sylow 2-subgroup of $M_{\mathbf{2 4}}$}

Now the vector space together with the upper uni-triangular matrices over $G F_{2}$ $(\langle b, c, d\rangle$ above) and the field automorphism $(\sigma)$, clearly generate a subgroup of shape $2^{6}:\left(D_{8} \times C_{2}\right)$ which must be a Sylow 2-subgroup of $M_{24}$. Indeed we see from the above that

$$
\left\langle\left[\begin{array}{ccc|c}
1 & a & b & \alpha \\
. & 1 & c & \beta \\
. & . & 1 & \gamma \\
\hline . & . & . & 1
\end{array}\right], \sigma \mid \begin{array}{l}
a, b, c \in G F_{2} \\
\alpha, \beta, \gamma \in G F_{4}
\end{array}\right\rangle \cong S y l_{2}\left(M_{24}\right)
$$

and the correspondence with permutations can be read off.

It should be noted that a Sylow 2-subgroup of $M_{24}$ consists precisely of those permutations preserving the octad, trio, sextet and pairing indicated in Fig. 7.

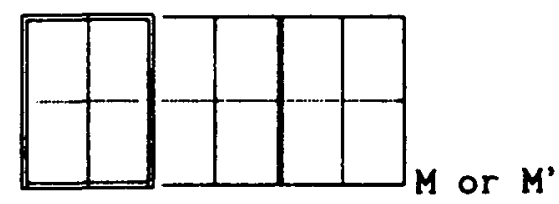

FIGURE 7

The Sylow 2-subgroup of $M_{24}$.

\section{REFERENCES}

1. D. J. Benson, The Construction of $J_{4}$ (Ph.D. thesis, Cambridge, 1980).

2. J. H. Conway, Three lectures on exceptional groups, in Finite Simple Groups (eds. M. B. Powell and G. Higman, Academic Press, London, Orlando and New York), 215-247.

3. J. H. Conway, The Miracle Octad Generator, in Topics in Group Theory and Computation (Ed. M. P. J. Curran, Academic Press, London, Orlando and New York, 1977), 62-68.

4. J. H. ConwaY, Hexacode and tetracode-MOG and MINIMOG, in Computational Group Theory (Ed. Michael D. Atkinson, Academic Press, London, Orlando and New York, 1984), 359-365.

5. R. T. Curtis, On subgroups of $\cdot 0$, I: lattice stabilizers, J. Algebra 27 (1973), 549-573. 
6. R. T. Curris, A new combinatorial approach to $M_{24}$, Math. Proc. Cambridge Philos. Soc. 79 (1976), 25-42.

7. R. T. Curtis, On subgroups of 0 , II local structure, J. Algebra 63 (1980), 413-434.

8. R. T. Curtis, Eight octads suffice, J. Combin. Theory Ser. A 36 (1984), 116-123.

9. J. A. TodD, A representation of the Mathieu group $\mathbf{M}_{24}$ as a collineation group, Anc. Mat. Para Appl. Ser. IV 71 (1966), 199-238.

10. R. A. Wilson, The maximal subgroups of Conway's group 2, J. Algebra 84 (1983), 107-114. 